\title{
Injection Drug Use as a Mediator Between Client-perpetrated Abuse and HIV Status Among Female Sex Workers in Two Mexico-US Border Cities
}

\author{
Monica D. Ulibarri - Steffanie A. Strathdee $\cdot$ Emilio C. Ulloa $\cdot$ \\ Remedios Lozada $\cdot$ Miguel A. Fraga - Carlos Magis-Rodríguez • \\ Adela De La Torre · Hortensia Amaro · Patricia O'Campo · Thomas L. Patterson
}

Published online: 28 July 2009

(c) The Author(s) 2009. This article is published with open access at Springerlink.com

\begin{abstract}
We examined relationships between clientperpetrated emotional, physical, and sexual abuse, injection drug use, and HIV-serostatus among 924 female sex workers (FSWs) in Tijuana and Ciudad Juarez, two large Mexico-US border cities. We hypothesized that FSWs' injection drug use would mediate the relationship between client-perpetrated abuse and HIV-seropositivity. The prevalence of client-perpetrated emotional, physical, and sexual abuse in the past 6 months was 26, 18, and $10 \%$ respectively; prevalence of current injection drug use and HIV was 12 and $6 \%$, respectively. Logistic regression analyses revealed that client-perpetrated sexual abuse was significantly associated with HIV-seropositivity and injection drug use, and that injection drug use was positively associated with HIV-seropositivity. Injection drug use partially mediated the relationship between client-perpetrated sexual abuse and HIV-seropositivity. Results suggest
\end{abstract}

M. D. Ulibarri · T. L. Patterson ( $\square)$

Department of Psychiatry, School of Medicine, University

of California, San Diego, 9500 Gilman Drive, La Jolla,

CA 92093-0680, USA

e-mail: tpatterson@ucsd.edu

M. D. Ulibarri

e-mail: mulibarri@ucsd.edu

S. A. Strathdee

Department of Medicine, School of Medicine, University

of California, San Diego, La Jolla, CA, USA

E. C. Ulloa

Department of Psychology, San Diego State University,

5500 Campanile Drive, San Diego, CA 92182-4611, USA

R. Lozada

Pro-COMUSIDA, Tijuana, BC, Mexico the need to address client-perpetrated violence and injection drug use when assessing HIV risk among FSWs.

Keywords Female sex workers .

Client-perpetrated abuse $\cdot$ Injection drug use $\cdot$ HIV

\section{Introduction}

This study examined the relationship between emotional, physical, and sexual abuse perpetrated by clients, injection drug use, and HIV-seropositivity among female sex workers (FSWs) in Tijuana and Ciudad (Cd.) Juarez, two large Mexico-US border cities where sex work is legally permitted within specific districts (usually referred to as the "Zona Roja" or red light district). Singer [1] has identified the co-occurrence of substance abuse, violence, and AIDS

\author{
M. A. Fraga \\ Facultad de Medicina y Psicología, Universidad Autónoma de \\ Baja California, Tijuana, BC, Mexico \\ C. Magis-Rodríguez \\ Centro Nacional para la Prevención y el Control del VIH/SIDA \\ (CENSIDA), Secretaría de Salud, Mexico, DF, Mexico
}

A. De La Torre

Chicana/o Studies, University of California, Davis, CA, USA

H. Amaro

Institute on Urban Health Research, Bouvé College of Health

Sciences, Northeastern University, Boston, MA, USA

P. O'Campo

Centre for Research on Inner City Health, University of Toronto,

Toronto, ON, Canada 
as the SAVA syndemic: mutually reinforcing connections between these conditions that disproportionately affect US inner-city populations living in poverty. Syndemics theory posits that endemic and epidemic conditions such as violence, substance abuse and AIDS in disadvantaged populations occur as a result of broader political, economic, and social factors [2]. Like the SAVA syndemic in the US, FSWs in Tijuana and Cd. Juarez are experiencing disproportionate rates of violence, substance abuse, and HIV/ AIDS compared to the larger Mexican population [3-5]. Tijuana and Cd. Juarez are economically depressed cities situated on major drug trafficking routes [6], making this region a nexus for violence, drug abuse, prostitution, sexual tourism, and rising rates of HIV and STIs.

HIV prevalence among FSWs in Tijuana and Cd. Juarez is significantly higher than that of the general Mexican adult population. HIV prevalence among FSWs in Tijuana was estimated to be $6 \%$ in 2005, with FSWs who inject drugs (FSW-IDUs) showing an even higher prevalence of $14 \%$ [7]. The national rate of HIV prevalence for Mexican adults is $0.3 \%$ [3]. In addition, the majority of FSWs in Tijuana and Cd. Juarez are low-income and report entering sex work out of economic necessity to support their family [8]. Thus, SAVA syndemic theory may provide a better understanding of the contextual factors that contribute to HIV risk among FSWs in the US-Mexico border region.

As suggested by Amaro over a decade ago [9], several studies have examined intimate partner violence (IPV) and history of abuse in relation to heterosexual women's HIV risk. Although an empirical understanding of the relationship between current experiences of violence and HIV infection is still emerging, Maman et al. [10] reviewed several theories that may explain why violence victimization predisposes women to contracting HIV: (1) through forced or coercive sexual intercourse with an HIV-infected partner; (2) by limiting women's ability to negotiate safe sexual behaviors; and (3) by establishing a pattern of sexual risk-taking among individuals assaulted in childhood and adolescence. More recently, studies have documented the association between IPV and HIV among heterosexual women in different international settings [11, 12] and age groups [13]. Specific risk behaviors associated with IPV include inconsistent condom use and increased likelihood of engaging in sex with an HIV-infected partner or an injection drug user (IDU) [14].

Ethnographic studies have documented the prevalence of history of abuse (i.e., history of childhood abuse, IPV, and client-perpetrated violence) and of HIV infection among FSWs in various international settings [15-17]. History of violence among FSWs has been associated with increased drug use and greater HIV vulnerability. For example, FSWs in India [18] who reported abuse from clients and intimate partners were less likely to use condoms and were therefore at increased sexual risk for HIV. In a study of FSWs in Canada, increased violence by clients and sex and drug-related HIV risk behaviors were associated with sharing illicit drugs with clients [16]. Surratt [19] reported that among FSWs in the US Virgin Islands, those who used illicit drugs were significantly more likely to report unprotected sex, client violence, and STIs. FSWs in New York City who injected drugs in the past year and who were HIV-positive were more likely to report combined physical and sexual abuse from intimate and commercial sexual partners [20]. Although these studies have identified specific HIV risk behaviors associated with histories of violence, few studies have focused on client-perpetrated violence specifically, and the causal links between violence and HIV-seropositivity have not been established.

Miller [21] developed a theoretical model in an effort to understand the relationship between history of sexual abuse and HIV risk among women. This model includes many of the sequelae associated with history of abuse including substance abuse, psychopathology (e.g., depression and PTSD), and sexual dissatisfaction and dysfunction (e.g., an inability to sustain intimate relationships, and participation in sex work). Miller proposed three possible causal pathways in the relation between sexual abuse and engaging in HIV-risky behavior: psychopathology as a result of abuse; drug use to cope with the abuse experience; and sexual adjustment problems related to taking sexual risks (e.g. obsession with sexual activity, participation in selfdestructive sexual relationships, and sex work). Based upon Miller's model, this study examines injection drug use as a mediator of the relationship between client-perpetrated violence and HIV-seropositivity among FSWs.

The specific links between history of abuse and HIVseropositivity may not be entirely clear and may not be unidirectional, but some research exists on the relationship between violence victimization and increased needle sharing among IDUs. In a multi-site study of female IDUs in the US, Wagner and colleagues [22] found that IPV was associated with higher levels of syringe sharing. In a study of IDUs in Vancouver, Canada, 68\% of women had a lifetime history of sexual violence, which was significantly associated with syringe sharing [23]. A previous study with IDUs in the same city showed that lifetime experience of sexual abuse was independently associated with syringe sharing among male and female IDUs [24]. However, these studies focused on lifetime experience of sexual violence, which could include either childhood or adulthood abuse, and results may not generalize to FSW populations currently experiencing abuse by clients. Therefore, further investigation of the relationship between recent clientperpetrated abuse, injection drug use, and HIV among FSWs is warranted. 
This study utilized cross-sectional data to test injection drug use as a mediator of the relationship between clientperpetrated abuse and HIV-seropositivity among FSWs. We hypothesized that: (1) client-perpetrated abuse would be associated with HIV-seropositivity; (2) client-perpetrated abuse would be associated with injection drug use by FSWs; and (3) injection drug use would be associated with HIV-seropositivity. Mediation analysis allows for the examination of possible mechanisms through which independent variables affect dependent variables [25]. We conducted a mediation analysis to examine injection drug use as a possible mechanism by which client-perpetrated abuse may affect HIV-serostatus, specifically examining whether injection drug use mediates the relationship between client-perpetrated abuse and HIV-seropositivity. Since only $3 \%(n=28)$ of FSW-IDUs in our sample reported sharing needles with their clients in the past 6 months, we chose to use injection drug use as the mediator instead of needle sharing.

\section{Methods}

\section{Participants}

Data were obtained from baseline interviews administered as part of an ongoing randomized trial of a safer sex intervention for FSWs in four Mexico-US border cities [26]. Baseline interview data from two of the four cities (Tijuana and Cd. Juarez) were available for use in this study. Participants were 924 FSWs from Tijuana and Cd. Juarez ( $n=474$ from Tijuana; $n=450$ from Cd. Juarez). The eligibility criteria for this study were: age $\geq 18$ years; traded sex for money, drugs, or other material benefits in the previous 2 months; and had unprotected sex with at least one client during the same time frame. Because one of the outcomes of the behavioral intervention was HIV incidence, FSWs who self-reported as HIV-positive at baseline were excluded from the study.

\section{Procedures}

Female sex workers (FSWs) residing in Tijuana and Cd. Juarez were recruited through street outreach, community and municipal health clinics, and referrals from participating FSWs. Interviews were conducted between January 2004 and March 2006. Women who completed the $60 \mathrm{~min}$, interviewer-administered survey received \$30 US dollars for their time. Study protocols were reviewed and approved by institutional review boards in San Diego, Tijuana, and Cd. Juarez.

\section{Measures}

The development of the questionnaire used in this study is described in detail elsewhere [27]. Client-perpetrated emotional, physical, and sexual abuse was assessed using three items selected from the family and social relationships section of the fifth edition of the Addiction Severity Index [28]. Participants responded either "yes" or "no" to whether they had experienced emotional, physical, and sexual abuse by a client in the past 6 months.

FSWs' injection drug use was assessed by a single "yes/ no" item asking participants if they injected drugs in the past 30 days.

HIV-status was ascertained using the Determine rapid HIV antibody test (Abbott Laboratories). Reactive samples were confirmed by EIA and Western blot. Pre- and posttest counseling was provided; participants with positive test results were referred to municipal health clinics for free medical care. Confirmatory HIV tests were conducted at either the San Diego County Health Department (for Tijuana samples) or the El Paso County Health Department (for Cd. Juarez samples).

\section{Data Analyses}

We compared FSWs who injected drugs in the past 30 days to those who did not, to identify any significant differences by IDU-status using Chi-square or Fisher's exact test for categorical variables and $t$-tests for continuous variables. Next, we conducted mediation analysis according to the four steps suggested by Baron and Kenny [25] utilizing a series of logistic regression analyses to determine the relationship between: (1) the independent variables (each of the client abuse variables) and the dependent variable (HIV-seropositivity); (2) the independent variables and the proposed mediator (injection drug use); and (3) the proposed mediator and the dependent variable. To examine mediation, a final logistic regression was run with both the independent variable and the proposed mediator as predictors of the dependent variable. For a full mediating effect to exist, the impact of the independent variable on the dependent variable must be zero after the mediator is included in the model. A partial mediating effect exists when the impact of the independent variable on the dependent variable is significantly reduced when the proposed mediator is included in the model. Finally, a Sobel test [29] was conducted to determine the significance of the indirect effect of the mediator. The Sobel test results in a zscore consisting of the indirect beta coefficient divided by the indirect standard error which tests whether the indirect effect of the independent variable on the dependent variable via the mediator is significantly different from zero. 


\section{Results}

\section{Descriptive Statistics}

The mean age of participants was 33.42 years $(\mathrm{SD}=9.11)$; average length of time as a FSW was 6.44 years $(\mathrm{SD}=6.74)$; and highest years of school completed was $6.14(\mathrm{SD}=3.15)$. In our sample, $6 \%(n=55)$ of the women were HIV-positive, and 12\% $(n=114)$ reported injecting drugs in the past 30 days. The mean number of male clients in the past 6 months was $336(\mathrm{SD}=325)$, and the median was 240 ( $\min .=1, \max .=1,920)$. Descriptive statistics for FSW-IDUs versus non-IDUs are presented in Table 1.

Prevalence of Abuse

In the total sample of FSWs, $26 \%$ reported experiencing emotional abuse by a client, $18 \%$ reported client-perpetrated physical abuse, and $10 \%$ reported client-perpetrated sexual abuse in the past 6 months. Among FSW-IDUs, 38\% reported client-perpetrated emotional abuse, $26 \%$ reported client-perpetrated physical abuse, and $21 \%$ reported clientperpetrated sexual abuse in the past 6 months (see Table 1).

\section{Bivariate Analyses}

Characteristics of IDU versus non-IDU FSWs in this study are presented in greater detail elsewhere [30]. However, client-perpetrated abuse was not previously examined. Briefly, FSW-IDUs were younger (30.98 vs. 33.77 years), had more male clients in the past 6 months (424.55 vs. 323.59), and differed with respect to marital status: FSWIDUs were more likely to be living with their romantic partner, while non-IDU FSWs were more likely to be single, separated, divorced, or widowed. FSW-IDUs were also more likely to have clients who had ever injected drugs (50 vs. 29\%) and to have experienced client-perpetrated emotional abuse (38 vs. 24\%), physical abuse (26 vs. $16 \%$ ), or sexual abuse ( 21 vs. $8 \%$ ) in the past 6 months, and to be HIV-positive (12 vs. 5\%). The specific values and significance levels of the test statistics are presented in Table 1.

\section{Mediation Analyses}

Since FSW-IDUs had significantly more clients overall and more IDU clients than non-IDU FSWs, these variables were controlled for in the logistic regression analyses. Of the three types of abuse by clients, sexual abuse was significantly associated with HIV-seropositivity ( $b=1.25$, $\mathrm{OR}=3.5, P<.001)$; whereas emotional and physical abuse were not. Hence, we conducted mediation analysis only for sexual abuse. As hypothesized, FSWs with higher rates of client-perpetrated sexual abuse were more likely to have injected drugs in the past 30 days $(b=1.09$, $\mathrm{OR}=3.0, P<.001$ ), and injection drug use (controlling for the effect of sexual abuse) was positively associated with HIV-seropositivity $(b=.82, \mathrm{OR}=2.26, P<.05)$.

Table 1 Sociodemographic and client abuse characteristics by IDU vs. non-IDU status $(N=924)$

\begin{tabular}{|c|c|c|c|c|c|c|c|c|c|c|}
\hline & \multicolumn{4}{|c|}{ Non-IDU $(n=810)$} & \multicolumn{4}{|c|}{ FSW-IDU $(n=114)$} & \multicolumn{2}{|c|}{ Test statistic } \\
\hline & $n$ & $\%$ & Mean & SD & $n$ & $\%$ & Mean & SD & $t$ & $X^{2}$ \\
\hline Age & & & 33.77 & 9.34 & & & 30.98 & 6.83 & $3.07 * *$ & \\
\hline Years of school completed & & & 6.07 & 3.15 & & & 6.65 & 3.09 & -1.80 & \\
\hline Years in sex work & & & 6.30 & 6.93 & & & 6.88 & 5.82 & -0.85 & \\
\hline Number of male clients (past 6 months) & & & 323.59 & 314.82 & & & 424.55 & 384.61 & $-3.11 * *$ & \\
\hline Has clients who have ever injected drugs & 235 & 29.0 & & & 57 & 50.0 & & & & $20.11 * * *$ \\
\hline Marital Status & & & & & & & & & & $23.58 * * *$ \\
\hline Married & 20 & 2.5 & & & 3 & 2.6 & & & & \\
\hline Living with partner & 157 & 19.4 & & & 42 & 36.8 & & & & \\
\hline Separated, Divorced, or Widowed & 228 & 28.1 & & & 15 & 13.9 & & & & \\
\hline Single & 401 & 49.5 & & & 53 & 46.5 & & & & \\
\hline Client-perpetrated emotional abuse (past 6 months) & 194 & 24.0 & & & 43 & 37.7 & & & & $9.83 * *$ \\
\hline Client-perpetrated physical abuse (past 6 months) & 133 & 16.4 & & & 30 & 26.3 & & & & $6.67 * *$ \\
\hline Client-perpetrated sexual abuse (past 6 months) & 64 & 7.9 & & & 24 & 21.1 & & & & $19.96 * * *$ \\
\hline HIV-Positive & 40 & 4.9 & & & 14 & 12.3 & & & & $9.79 * *$ \\
\hline
\end{tabular}

Note: Some frequencies do not sum to $N$ due to missing values

* Significant at $P<.05$

** Significant at $P<.01$

*** Significant at $P<.001$ 


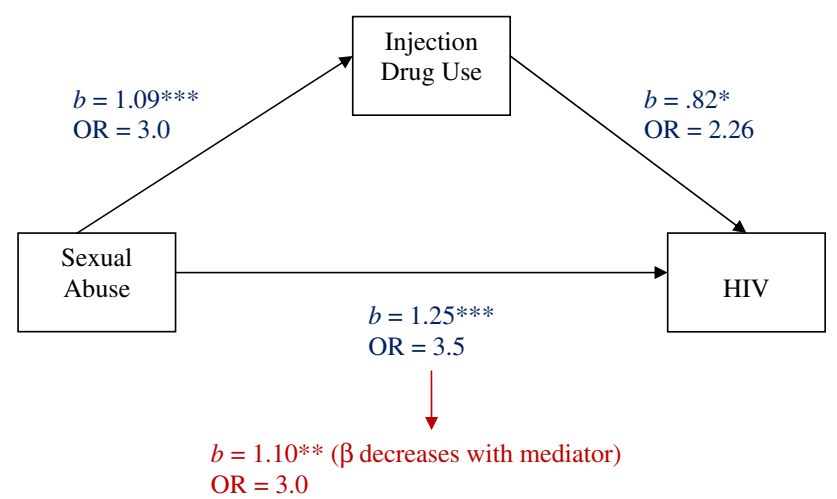

Fig. 1 FSWs injection drug use mediating the relationship between client-perpetrated sexual abuse and HIV-status. $* P<.05$, *** $P<.01, * * * P<.001$

FSWs' injection drug use partially mediated the relationship between client-perpetrated sexual abuse and HIVseropositivity. When both client-perpetrated sexual abuse and FSWs' injection drug use were regressed simultaneously onto HIV-seropositivity, there was a decrease in the relationship between sexual abuse and seropositivity from $b=1.25, \quad \mathrm{OR}=3.5, \quad P<.001$ to $b=1.10$, $\mathrm{OR}=3.0, P<.01$ (see Fig. 1). An additional Sobel's test of the indirect effect confirmed the significance of the mediator $(z=2.05, P<.05)$.

Intimate partner violence (IPV), childhood physical and sexual abuse, and unprotected vaginal and anal sex with clients were examined as potential confounds. FSW-IDUs did not differ from non-IDUs with respect to IPV, but they were more likely to have experienced childhood physical and sexual abuse. However, childhood physical and sexual abuse were not significantly associated with HIV status ( $r=.06$ and .02 , respectively); therefore, they were not controlled for in the regression analyses. FSW-IDUs did not differ from non-IDUs with respect to unprotected sex with clients, nor was this variable significantly associated with HIV status [30, 31]; therefore, it was deemed not necessary to control for this variable in the regression analyses, and injection drug use remained the variable of focus for mediation.

\section{Discussion}

Consistent with previous studies of FSWs, the women in our sample reported frequent experience of client-perpetrated abuse. Moreover, FSW-IDUs were significantly more likely to experience client-perpetrated emotional, physical, and sexual abuse and be HIV-positive as compared to their nonIDU counterparts. Being an IDU may be a critical marker in identifying FSWs who are at increased risk for violence victimization and infection with HIV. Our results showed that client-perpetrated abuse was significantly associated with a higher prevalence of injection drug use among FSWs, and those who experienced client-perpetrated sexual abuse were more likely to be HIV-positive, highlighting the need to consider history of abuse and injection drug use when assessing HIV risk among FSWs in this region.

As hypothesized, injection drug use partially mediated the relationship between client-perpetrated sexual abuse and HIV-status, suggesting that injection drug use may account for a significant proportion of this relationship. FSWs who are victims of violence may engage in drug use in order to numb themselves from the psychological consequences of the abuse and fear of revictimization. Wechsberg et al. [17] found that sex workers in South Africa with a history of abuse (childhood, IPV, and clientperpetrated) had a fear of future victimization and discomfort with the relationship dynamics with men, which in turn led to increased substance abuse and greater sexual risk. Additionally, FSWs may be more likely to acquiesce to requests for unsafe sex in order to earn more money to support their drug addiction [30].

Although this study identified injection drug use and history of client-perpetrated violence as important correlates of HIV risk among Mexican FSWs, it is likely that there is an underlying complexity of risk factors such as mental health disorders associated with trauma, gendered power dynamics, poverty, and societal norms and practices that contribute to FSWs' overall risk [32-34]. Furthermore, these risks may be exacerbated by injection drug use. For example, a qualitative study among male and female IDUs in Tijuana and Cd. Juarez found that female IDUs often obtained and used drugs within the context of a sexual relationship with a drug-using, male romantic partner or spouse [35]. This could contribute to women's receptive needle sharing or being "second on the needle," [35, 36] which in turn may reflect a form of submission to their male partners [37]. These observations suggest that research with FSW-IDUs and their drug and sexual partners should take into consideration relationship dynamics and the intertwined nature of their drug use with sexual partnerships. Further research is warranted examining multi-level (i.e., micro-, meso-, and macro-level) factors of risk among this population. HIV prevention research targeting both FSWs and clients is urgently needed in order to adequately account for the clients' sexual and drug risk taking and to identify potentially overlapping networks of high-risk drugand sex-related partners who can be targeted for HIV prevention and harm reduction efforts [16, 38].

Although this study adds to the knowledge base on the relationship between client-perpetrated abuse, drug use, and HIV risk among FSWs, several limitations must be noted. First, because this study was cross-sectional, causal inferences cannot be drawn. Additional longitudinal studies 
that assess onset of abuse, injection drug use, and HIVseropositivity may further clarify the observed associations. Second, the present study is retrospective and relies primarily on self-report data, which can entail recall bias. However, the recall period for the questions was relatively short (e.g., past 30 days to past 6 months), and previous research has provided validation for self-report data with IDU populations [39-41]. A third limitation was the limited, fixed nature of the abuse items selected from the Addiction Severity Index. These items did not assess multiple experiences of abuse, extent of abuse, or the number of different abusers, and therefore it may not reflect the true experience of client-perpetrated abuse for some of the FSWs in our study. Last, the results of this study may not be generalizable to other ethnic groups of FSWs, nor even to other Latina FSW groups. The participants in this study constituted convenience samples from two US-Mexico border cities and may not be representative of FSWs in other cities or of FSWs in either of those cities as a whole, especially since the intervention study deliberately recruited high-risk FSWs.

Further understanding of the relationship between client-perpetrated violence, injection drug use by FSWs, and FSWs' HIV-risk behavior is important for the development of treatment and prevention strategies that adequately address the complexity of risks that FSW-IDUs face. Future HIV prevention interventions serving FSWs in this region should include violence screening and prevention as well as treatment for mental disorders, in order to address any negative sequelae of trauma and to help FSWs avoid potentially violent clients. The results of this study also suggest the need to treat victimized FSWs' injection drug use, their impairment due to drug use, or both, in order to reduce the effect of client-perpetrated abuse on the risk of acquiring HIV. Increased access to drug treatment services may greatly enhance HIV prevention for this population. For example, Amaro and colleagues [42] found that treatment of drug addiction and trauma simultaneously was effective in reducing HIV sex-risk behaviors among poor inner-city Latina and African American women.

Finally, there is a need for HIV prevention interventions to move beyond exclusively individual-level approaches by including male clients in order to reduce women's vulnerability to violence and HIV risks. A solely individual-level approach assumes that women can engage in preventive behaviors free from influence from their partners or larger societal norms [33]. However, research indicates that women's HIV risk behavior is influenced by partner violence and power relationships that impact their ability to negotiate sexual and drug risk reduction behaviors [16-18, 22].

Acknowledgments The authors respectfully acknowledge the participation of all the women in this study for making this work possible. We also thank the US-Mexico bi-national study staff, Brian Kelly and Sandra Volz for editorial assistance, and Dr. Willo Pequegnat at the National Institute of Mental Health for her support and encouragement. Funding for this research was provided through NIMH Grants R01 MH65849, R01 MH65849-Supp, and NIDA Grant R01 DA023877.

Open Access This article is distributed under the terms of the Creative Commons Attribution Noncommercial License which permits any noncommercial use, distribution, and reproduction in any medium, provided the original author(s) and source are credited.

\section{References}

1. Singer M. A dose of drugs, a touch of violence, a case of AIDS: conceptualizing the SAVA syndemic. Free Inq Creat Sociol. 1996;24(2):99-110.

2. Singer M. AIDS and the health crisis of the US urban poor: the perspective of critical medical anthropology. Soc Sci Med. 1994;39(7):931-48.

3. CENSIDA. Panorama Epidemiologico del VIH/SIDA e ITS en Mexico: centro Nacional para la Prevencion y Control del VIH/ SIDA2006 June 302006.

4. Secretaria de Salud. Encuesta Nacional de Adicciones. Available at. www.salud.gob.mx/unidades/cdi/documentos/DOCSAL7326. pdf. Accessed April 152008.

5. US Department of State. Travel Alert. Available at: http://travel.state.gov/travel/cis_pa_tw/pa/pa_3028.html. Accessed March 12009.

6. Bucardo J, Brouwer KC, Magis-Rodriguez C, Ramos R, Fraga M, Perez SG, et al. Historical trends in the production and consumption of illicit drugs in Mexico: implications for the prevention of blood borne infections. Drug Alcohol Depend. 2005;79(3):281-93.

7. Strathdee SA, Lozada R, Semple SJ, Orozovich P, Pu M, StainesOrozco H, et al. Characteristics of female sex workers with US clients in two Mexico-US border cities. Sex Transm Dis. 2008;35(3):263-8.

8. Bucardo J, Semple SJ, Fraga-Vallejo M, Davila W, Patterson TL. A qualitative exploration of female sex work in Tijuana, Mexico. Arch Sex Behav. 2004;33(4):343-51.

9. Amaro H. Love, sex, and power: considering women's realities in HIV prevention. Am Psychol. 1995;50(6):437-47.

10. Maman S, Campbell J, Sweat MD, Gielen AC. The intersections of HIV and violence: directions for future research and interventions. Soc Sci Med. 2000;50(4):459-78.

11. Gonzalez-Guarda RM, Peragallo N, Urrutia MT, Vasquez EP, Mitrani VB. HIV risks, substance abuse, and intimate partner violence among Hispanic women and their intimate partners. J Assoc Nurses AIDS Care. 2008;19(4):252-66.

12. Silverman JG, Decker MR, Saggurti N, Balaiah D, Raj A. Intimate partner violence and HIV infection among married Indian women. JAMA. 2008;300(6):703-10.

13. Sormanti M, Shibusawa T. Intimate partner violence among midlife and older women: a descriptive analysis of women seeking medical services. Health Soc Work. 2008;33(1):33-41.

14. El-Bassel N, Gilbert L, Wu E, Chang M, Gomes C, Vinocur D, et al. Intimate partner violence prevalence and HIV risks among women receiving care in emergency departments: implications for IPV and HIV screening. Emerg Med J. 2007;24(4):255-9.

15. Sanders T. Female street workers, sexual violence, and protection strategies. J Sex Aggress. 2001;7(1):5-18.

16. Shannon K, Kerr T, Bright V, Gibson K, Tyndall MW. Drug sharing with clients as a risk marker for increased violence and 
sexual and drug-related harms among survival sex workers. AIDS Care. 2008;20(2):228-34.

17. Wechsberg WM, Luseno WK, Lam WK. Violence against substance-abusing South African sex workers: intersection with culture and HIV risk. AIDS Care. 2005;17:S55-64.

18. Panchanadeswaran S, Johnson SC, Sivaram S, Srikrishnan AK, Latkin C, Bentley $\mathrm{ME}$, et al. Intimate partner violence is as important as client violence in increasing street-based female sex workers' vulnerability to HIV in India. Int $\mathbf{J}$ Drug Policy. 2008;19(2):106-12.

19. Surratt H. Sex work in the Caribbean Basin: patterns of substance use and HIV risk among migrant sex workers in the US Virgin Islands. AIDS Care. 2007;19(10):1274-82.

20. El-Bassel N, Witte SS, Wada T, Gilbert L, Wallace J. Correlates of partner violence among female street-based sex workers: substance abuse, history of childhood abuse, and HIV risks. AIDS Patient Care STDS. 2001;15(1):41-51.

21. Miller M. A model to explain the relationship between sexual abuse and HIV risk among women. AIDS Care. 1999;11(1):3-20.

22. Wagner KD, Hudson SM, Latka MH, Strathdee SA, Thiede H, Mackesy-Amiti ME, et al. The effect of intimate partner violence on receptive syringe sharing among young female injection drug users: an analysis of mediation effects. AIDS Behav. 2009;13:217-24.

23. Braitstein P, Li K, Tyndall M, Spittal P, O’Shaughnessy MV, Schilder A, et al. Sexual violence among a cohort of injection drug users. Soc Sci Med. 2003;57(3):561-9.

24. Strathdee SA, Patrick DM, Archibald CP, Ofner M, Cornelisse PG, Rekart M, et al. Social determinants predict needle-sharing behaviour among injection drug users in Vancouver, Canada. Addiction. 1997;92(10):1339-47.

25. Baron RM, Kenny DA. The moderator-mediator variable distinction in social psychological research: conceptual, strategic, and statistical considerations. J Pers Soc Psychol. 1986;51:117382.

26. Patterson TL, Semple SJ, Fraga M, Bucardo J, De la Torre A, Salazar-Reyna J, et al. A sexual risk reduction intervention for female sex workers in Mexico: design and baseline characteristics. J HIV AIDS Soc Serv. 2006;5(2):115-37.

27. Patterson TL, Semple SJ, Fraga M, Bucardo J, Davila-Fraga W, Strathdee SA. An HIV-prevention intervention for sex workers in Tijuana, Mexico: a pilot study. Hisp J Behav Sci. 2005;27(1):82100.

28. McLellan AT, Kushner H, Metzger D, Peters R. The fifth edition of the addiction severity index. J Subst Abuse Treat. 1992; 9(3):199-213.

29. Sobel ME. Asymptotic confidence intervals for indirect effects in structural equations models. In: Leinhart S, editor. Sociological methodology. San Francisco: Jossey-Bass; 1982. p. 290-312.
30. Strathdee SA, Philbin MM, Semple SJ, Pu M, Orozovich P, Martinez G, et al. Correlates of injection drug use among female sex workers in two Mexico-US border cities. Drug Alcohol Depend. 2008;92(1-3):132-40.

31. Patterson TL, Semple SJ, Staines H, Lozada R, Orozovich P, Philbin MM, et al. Prevalence and correlates of HIV infection among female sex workers in two Mexico-US border cities. J Infect Dis. 2008;197:728-32.

32. Choi SYP, Holroyd E. The influence of power, poverty and agency in the negotiation of condom use for female sex workers in mainland China. Cult Health Sex. 2007;9(5):489-503.

33. Shannon K, Kerr T, Allinott S, Chettiar J, Shoveller J, Tyndall MW. Social and structural violence and power relations in mitigating HIV risk of drug-using women in survival sex work. Soc Sci Med. 2008;66(4):911-21.

34. Surratt HL, Kurtz SP, Weaver JC, Inciardi JA. The connections of mental health problems, violent life experiences, and the social milieu of the 'stroll' with the HIV risk behaviors of female street sex workers. J Psychol Hum Sex. 2005;17(1):23-44.

35. Cruz MF, Mantsios A, Ramos R, Case P, Brouwer KC, Ramos $\mathrm{ME}$, et al. A qualitative exploration of gender in the context of injection drug use in two US-Mexico border cities. AIDS Behav. 2007;11(2):253-62.

36. Harvey E, Strathdee SA, Patrick DM, Ofner M, Archibald CP, Eades G, et al. A qualitative investigation into an HIV outbreak among injection drug users in Vancouver, British Columbia. AIDS Care. 1998;10(3):313-21.

37. Andrade R, Estrada AL. Are Hispana IDUs Tecatas?: reconsidering gender and culture in Hispana injection drug use. Subst Use Misuse. 2003;38(8):1133-58.

38. Strathdee SA, Sherman SG. The role of sexual transmission of HIV infection among injection and non-injection drug users. J Urban Health. 2003;80(4 (suppl 3)):iii7-14.

39. Darke S, Hall W, Heather N, Ward J. The reliability and validity of a scale to measure HIV risk-taking behaviour among intravenous drug users. AIDS. 1991;5(2):181-5.

40. De Irala J, Bigelow C, McCusker J, Hindin R, Zheng L. Reliability of self-reported human immunodeficiency virus risk behaviors in a residential drug treatment population. Am J Epidemiol. 1996;143(7):725-32.

41. Van Duynhoven YT, Nagelkerke NJ, Van De Laar MJ. Reliability of self-reported sexual histories: test-retest and interpartner comparison in a sexually transmitted diseases clinic. Sex Transm Dis. 1999;26(1):33-42.

42. Amaro H, Larson MJ, Zhang A, Acevedo A, Dai J, Matsumoto A. Effects of trauma intervention on HIV sexual risk behaviors among women with co-occurring disorders in substance abuse treatment. J Commun Psychol. 2007;35(7):895-908. 